\title{
Interorganisationales Kostenmanagement - Erfolgspotenzial oder Kooperationsrisiko?
}

\author{
Klaus Möller · Felix Isbruch
}

Published online: 18 January 2008

(C) The Author(s) 2008

Zusammenfassung Interorganisationales Kostenmanagement (IOCM) stellt einen Ansatz dar, um in der Wertschöpfungskette Kostenoptimierungen über Unternehmensgrenzen hinweg zu erschließen. Zahlreiche Fallstudien zeigen, dass IOCM eine partnerschaftliche Zusammenarbeit der beteiligten Unternehmen erfordert und erfolgssteigernd wirken kann. Der Beitrag untersucht im Rahmen einer großzahligen Befragung anhand eines Strukturgleichungsmodells, inwieweit der Beziehungskontext ausschlaggebend für den Erfolg des IOCM ist. Basierend auf Aussagen der Transaktionskosten-, Konflikt- und Austauschtheorie wird gezeigt, dass IOCM nur unter bestimmten Voraussetzungen erfolgssteigernd wirkt. Die Daten basieren auf einer Erhebung bei 164 Automobilzulieferern aus Deutschland.

Schlüsselwörter Beziehungskontext · Empirische Forschung · Erfolg · Interorganisationales Kostenmanagement · Kooperation · Zulieferer

Summary Interorganizational cost management (IOCM) is a widespread approach to reduce costs throughout different organizations in the supply chain. Numerous case studies showed that a culture of partnership is required to secure a successful use of IOCM. Based on a large scale empirical study using structural equation modeling we research the relational context and its impact on IOCM. Drawing on transaction cost theory, conflict theory and social exchange theory, this paper shows evidence that IOCM is only under certain relational circumstances successful. The data are based on information of 164 German automotive suppliers.

Prof. Dr. K. Möller (凶) · Dipl.-Kfm. F. Isbruch

Professur für Unternehmensrechnung und Controlling, Georg-August-Universität Göttingen,

Platz der Göttinger Sieben 3, 37073 Göttingen, Deutschland

e-mail: Klaus.Moeller@wiwi.uni-goettingen.de

e-mail: Felix.Isbruch@wiwi.uni-goettingen.de 
Keywords Relational context · Empirical study · Performance interorganizational cost management $\cdot$ Cooperation supply chain

\section{Einleitung}

In der Folge einer zunehmend arbeitsteiligen Leistungserstellung entstehen einerseits immer stärker spezialisierte Organisationen, andererseits immer stärkere Leistungsverflechtungen zwischen diesen Organisationen (Picot et al. 2003, S.2ff.). Der Verbesserung der Zusammenarbeit zwischen den Organisationseinheiten widmet sich das Kooperationscontrolling, das sein Hauptaugenmerk in industriellen Geschäftsbeziehungen insbesondere auf die vertikale Zusammenarbeit in Supply Chains legt. (Stölzle 1999, Stölzle und Otto 2003, Seuring und Goldbach 2002). Fokussiert man weiter auf die proaktive Gestaltung der in einer Kooperation entstehenden Kosten haben sich verschiedene Ansätze etabliert, die unter dem Begriff interorganisationales Kostenmanagement bzw. Inter Organisational Cost Management (IOCM) diskutiert werden (Cooper und Slagmulder 1999, Dekker 2004). Ziel ist es, die in den verschiedenen Wertschöpfungsstufen anfallenden Kosten über die Unternehmensgrenzen hinweg weiter zu optimieren. Derartige Maßnahmen werden in der Wissenschaft rege diskutiert und in der Unternehmenspraxis häufig eingesetzt (Dekker 2003, Anderson 2007, Hakanson und Lind 2007). In beiden Feldern fällt eine durchaus kontroverse Diskussion auf: Einerseits werden dem IOCM große Einsparpotenziale zugeschrieben, andererseits werden seine Einsatzmöglichkeiten kritisch gesehen. Pro- und Contra-Argumente stammen sowohl aus der Theorie (bspw. Reduzierung von Transaktionskosten einerseits, potenzielle Verteilungs- und Rollenkonflikte andererseits) als auch der Praxis (bspw. bessere Zusammenarbeit einerseits, Ausnutzung von Abhängigkeiten durch mächtige Kunden andererseits). Auffällig ist, dass es sich ausschließlich um die Darstellung von Einzelfallstudien handelt, deren Generalisierbarkeit meist auch von den Autoren kritisch beurteilt wird (Cooper und Yoshikawa 1994, S. 52, Carr und Ng 1995, S. 359, Mouritsen et al. 2001, S. 238, Dekker 2003, S. 19, Cooper und Slagmulder 2004, S.24, Kulmala 2004, S.67). Ziel des vorliegenden Beitrags ist es daher, im Rahmen einer großzahligen Überprüfung einerseits die Erfolgsrelevanz des IOCM zu überprüfen und andererseits beziehungsorientierte Einflussfaktoren auf die Wirkung des IOCM zu identifizieren. Als Grundlage dient eine Stichprobe von deutschen Automobilzulieferern, die im Rahmen einer Befragung im Jahr 2006 erhoben wurde. Adressiert wurden sämtliche 733 Automobilzulieferer in Deutschland. Antworten wurden von 164 Unternehmen ausgewertet, was einer guten Rücklaufquote von 22,2\% entspricht. Methodisch findet ein Strukturgleichungsmodell Anwendung. Die Automobilzulieferindustrie zeichnet sich durch eine ,close but adversarial“ Situation (Mudambi und Helper 1998, S. 790) bzw. eine große Abhängigkeit, hohen Kostendruck sowie Enge der Zusammenarbeit aus. Sie erscheint daher besonders geeignet für die Überprüfung der o. a. Fragestellung.

Der Beitrag gliedert sich dazu in fünf Abschnitte: Im folgenden, zweiten Abschnitt wird zuerst der Stand der Forschung zum IOCM dargestellt. In Kapitel drei wird an- 
schließend der Bezugsrahmen der Untersuchung entwickelt, der theoretische Ansätze im IOCM sowie die zentralen Einflussfaktoren umfasst. Kapitel vier gibt dann die Ergebnisse der empirischen Untersuchung wieder, die kritisch diskutiert werden. Der Beitrag schließt mit einem Fazit.

\section{Interorganisational Cost Management (IOCM)}

Im Gegensatz zur dokumentierenden und damit vergangenheitsorientierten Kostenrechnung bezeichnet der Begriff Kostenmanagement die aktive und zielorientierte Gestaltung und Beeinflussung von Kostenstruktur, -niveau und -verlauf (Kajüter 2000, Franz und Kajüter 2002). Damit sind Entscheidungen im Kontext des Kostenmanagements immer zukunftsorientiert (z. B. Produktdifferenzierung, Fertigungstiefe, Produktionsverfahren, Logistik). Das Kostenmanagement wird dazu notwendigerweise in frühen Phasen der Produktentwicklung und des Produktanlaufs gestaltend tätig, um die Kostenverursachung zielorientiert zu steuern. Wesentliches Element des Kostenmanagements ist seine Funktion als Impulsgeber, um Unwirtschaftlichkeiten und Gestaltungsspielräume zu einem frühen Zeitpunkt und in den richtigen Bereichen antizipativ aufzuzeigen. Überträgt man diese Ansätze auf die Zusammenarbeit zwischen Hersteller/Original Equipment Manufacturer (OEM) und Zulieferer, ergibt sich das IOCM. Dazu findet während des Entwicklungsprozesses ein Informationsaustausch zwischen beiden statt, indem gemeinsam Potentiale zur Kostenreduzierung anhand der Kundenspezifikationen erarbeitet werden (Cooper und Slagmulder 2004, S. 3ff., Kajüter 2002, S. 42). ,,The key extension of IOCM [...] is the active involvement of both the buyer's and supplier's design teams in the joint management of costs." (Cooper und Slagmulder 2004, S. 6). IOCM fördert damit Innovationen und schafft so einen Wettbewerbsvorteil für die beteiligten Unternehmen (Cooper und Yoshikawa 1994, S. 52). Cooper und Slagmulder als zentrale Protagonisten der IOCM-Forschung definieren IOCM als , ... structured approach to coordinating the activities of firms in a supplier network so that total costs in the network are reduced“ (Cooper und Slagmulder 1999, S. 145f.). Instrumentell gestalten sie das IOCM mittels vier Ansätzen aus: (Cooper und Slagmulder 1999, S. 147-151, ähnlich Seuring 2001, S. 70-84):

- Chained Target Costing (CTC): Zerlegung des marktseitig durchsetzbaren Preises für das Endprodukt auf die einzelnen Komponenten und Wertschöpfungsstufen. Im Gegensatz zum ,einfachen“ Target Costing beteiligen sich beim Chained Target Costing alle beteiligten Partner aktiv an der Erreichung des Target Price.

- Functionality-Price-Quality Technique: Bei geringeren Abweichungen soll die Kosteneinsparung durch eine Einschränkung der Funktionalität oder Qualität (FPQ) erreicht werden.

- Interorganizational Cost Investigations (ICI): Gemeinsame wertanalytische Untersuchungen von Hersteller und Zulieferer zur Kostenoptimierung und entsprechendes Redesign des Produktes. Hierunter ist auch das sogenannte Open Book Accounting, also der Austausch von Kalkulationsinformationen zu subsumieren (Kajüter und Kulmala 2005, S. 180). 
- Concurrent Cost Management (CCM): Interorganisationale Projektteams stellen über das ICI hinaus den gesamten Wertschöpfungsprozess in Frage, wobei bereits in frühen Produktentwicklungsphasen miteinander kooperiert wird.

Nicht nur Cooper und Slagmulder gehen dabei von einem direkten Zusammenhang zwischen dem Einsatz von IOCM und der Steigerung des Unternehmenserfolgs aus (Cooper und Slagmulder 1999, S. 145f., Mouritsen et al. 2001, S. 222, Kajüter 2002, S.42). Weitere Untersuchungen auf Basis kontingenztheoretischer Überlegungen zeigen jedoch, dass der Beziehungskontext entscheidend für den Anwendungserfolg und die Art der Nutzung des IOCM ist (Cooper und Slagmulder 2004, S.20f.). Hierbei wurden das Ausmaß gemeinsam entwickelter Produkte, die Ressourcenteilung, der Grad der Lieferantenintegration, gegenseitiges Commitment und informelle Schutzmechanismen als zentrale Einflussfaktoren identifiziert. Je weiter die gemeinsame Produktentwicklung vorangetrieben ist und je mehr sich die Verantwortlichkeiten zwischen Hersteller und Zulieferer aufteilen, desto höher muss demnach die Integration beider Partner hinsichtlich Entwicklungs- und Herstellungsstandards sein. Auch die Erstellung und Teilung gemeinsamer Ressourcen ist Teil des Kontextes. Diese kooperationsspezifischen Investitionen verlieren außerhalb der Kooperation ihren Wert und wirken durch die so entstehenden Wechselkosten als Austrittsbarriere (Dyer und Singh 1998, S.662, Mudambi und Helper 1998, S.786). Auch im Bereich der Lieferantenintegration lassen sich verschiedene Stufen identifizieren: Je früher die Lieferanten in den Entwicklungsprozess mit einbezogen werden, je bedeutsamer die an sie ausgelagerten Produktionsprozesse für den Hersteller sind und je mehr Verantwortlichkeiten sie in diesen Prozessen übernehmen, desto enger und besser wird das Verhältnis von Hersteller und Lieferant (Cooper und Slagmulder 2004, S. 13f., Möller 2002, S. 60f.). Mit zunehmender Integration ist auch eine Steigerung des gegenseitigen Commitments durch Stabilisierung der Geschäftsbeziehung über längere Zeit und eine engere Zusammenarbeit verbunden, diese wird durch die Bindungswirkung gemeinsamer Ressourcen weiter gestärkt. Aus den genannten Merkmalen entstehen dann mit zunehmender gegenseitiger „Vertrautheit“, wiederholter Interaktion und kalkuliertem Verhalten informelle, auf Vertrauen und Beziehungsnormen basierende Schutzmechanismen der Zusammenarbeit (Adler 2001, S.218, Gundlach et al. 1995, S. 78). Zwischen den vorgenannten Komponenten des Beziehungskontextes und den Möglichkeiten zur Implementierung und dem Erfolg von IOCM wurde anhand von Einzelfallstudien ein direkter Zusammenhang nachgewiesen (Cooper und Slagmulder 2004, S. 20f.)

Unter dem Begriff des IOCM soll im Folgenden die systematische und formalisierte, unternehmensübergreifende Identifikation und Realisierung von Kosteneinsparpotenzialen verstanden werden, die in Teams von allen an der Kooperation beteiligten Partnerunternehmen betrieben wird (Cooper und Yoshikowa 1994, S. 54, Cooper und Slagmulder 1999, S. 145). Unternehmenskooperationen stellen dabei eine vertraglich vereinbarte Zusammenarbeit mindestens zweier rechtlich selbstständiger Partnerunternehmen zur gemeinsamen Erstellung von Produkten oder Dienstleistungen dar (Balling 1997, S. 13ff., Möller 2006, S.62). 


\section{Bezugsrahmen der Untersuchung}

Im folgenden Abschnitt sollen die wesentlichen theoretischen Grundlagen des IOCM dargestellt werden: Neue Institutionenökonomie, soziale Austauschtheorie sowie Konflikttheorie. Sie sind die Grundlage für die Analyse des Kontextes der kooperativen Beziehung und determinieren damit den Erfolg des IOCM. Aus der theoretischen Analyse lassen sich zentrale Sachverhalte ableiten, die Eingang in die empirische Untersuchung finden und daher gleichfalls erläutert werden.

\subsection{Theoretische Ansätze im IOCM}

\subsubsection{Neue Institutionenökonomie}

Die Transaktionskostentheorie basiert auf der Überlegung, dass institutionelle Organisationsformen durch die Entstehung von Kosten bei der Koordination wirtschaftlicher Aktivitäten zu erklären sind (Coase 1937, S. 386ff., Williamson 1975, S. 38). Transaktionskosten werden häufig mit Koordinationskosten gleichgesetzt und umfassen neben den (monetär bewertbaren) Informations- und Kommunikationskosten zur Durchführung einer Transaktion auch qualitative nachteilige Komponenten wie Anstrengung, Zeitaufwand sowie die Kosten für Mechanismen zur Opportunismusvermeidung etc. (Picot et al. 1999, S. 67). Die Auswahl und Beurteilung der effizientesten institutionellen Koordinationsform, d. h. ob eine Leistung intern zu erbringen (Hierarchie), von einem externen Zulieferer zu beziehen (Markt) oder im Rahmen eines hybriden Arrangements (weitergehende Kooperation mit einem Zulieferer) zu erstellen ist erfolgt durch einen Vergleich der Transaktionskosten (Picot 1982, S. 273). Zulieferkooperationen entstehen, wenn die Transaktionskosten durch eine hybride Koordination am geringsten sind. Zulieferkooperationen sind darüber hinaus in hohem Maße durch gegenseitige Informationsasymmetrien gekennzeichnet. Der Zulieferer verfügt über detailliertere Informationen über die genaue Kostenzusammensetzung der hergestellten Komponenten, der OEM-Hersteller dagegen kann besser den am Markt durchsetzbaren Preis einschätzen. Es besteht somit eine klassische, gegenseitige Prinzipal-Agenten Beziehung. Beide Partner können ihren jeweiligen Informationsvorsprung zu ihrem eigenen Vorteil ausnutzen und sich zueinander opportunistisch verhalten, etwa wenn der Zulieferer die entstandenen Kosten zu hoch angibt, um höhere Preise durchsetzen zu können.

\subsubsection{Soziale Austauschtheorie}

Die soziale Austauschtheorie befasst sich damit, inwieweit das Verhalten eines Akteurs das eines anderen verstärken oder abschwächen kann. Damit folgt diese Theorie einem behavioristischen Menschenbild. Der Akteur versucht, den positiven Nutzen seiner Handlungen zu maximieren und negative Effekte $\mathrm{zu}$ minimieren (Thibaut und Kelley 1959, S.49, Blau 1964). Rusbult integrierte das Commitment in die soziale Austauschtheorie. Die Bindung entsteht in diesem Modell durch beziehungsspezifische Investitionen, die von einem Wechsel abhalten. Die Bindung wird um so höher, je größer die Zufriedenheit ist und je kleiner die Attraktivität der ande- 
ren Optionen ist (Rusbult 1983, S. 431ff., Wieselquist et al. 1999, S. 943, Das und Teng 2002, S. 447-449). Im Falle des IOCM stärken kooperationsspezifische Investitionen die Bindung der Partner an die Zusammenarbeit. So ist die steigende Zuliefererintegration in den Entwicklungs- und Produktionsprozess eine beziehungsspezifische Investition, die die Bindung des OEM-Herstellers an den Lieferanten erhöht.

\subsubsection{Konflikttheorie}

Die Konflikttheorie unterscheidet verschiedene Konfliktursachen für den Fall der Kooperation, zu nennen sind vertikale Verteilungsbeziehungen, Zielbeziehungen, Rollenbeziehungen, Machtbeziehungen und Informationsbeziehungen, deren Zusammenspiel die Konfliktfelder beschreibt (Steffenhagen 1975, S. 72ff., Meinig und Heß 1992, S. 373, Pampel 1993, S. $211 \mathrm{ff}$.). Unter Verteilungskonflikte fällt auch die Forderung, dass die Teilnahme an der Kooperation für jeden Teilnehmer einen positiven Nettonutzen erbringen muss. Rollenkonflikte können bei Abweichungen von den klassischen Rollenerwartungen entstehen, die im Bereich des Lieferanten etwa die Rechtfertigung des Vertrauens auf Geheimhaltung, im Bereich des Herstellers die Lieferantentreue und kooperatives Verhalten beinhalten. Informationskonflikte dagegen entstehen aus der asymmetrischen Verteilung von Informationen hinsichtlich Fertigungs-Knowhow oder Absatzchancen (Pampel 1993, S. 218f.). Krystek et al. heben zudem Konfliktpotenziale bei der Verteilung der Aufgaben und Kosten für interorganisationale Informationssysteme, wie sie Voraussetzung für das IOCM sind, hervor (Krystek et al. 1997, S. 272f.).

\subsection{Zentrale Einflussfaktoren und Messkonstrukte}

Basierend auf den gerade vorgestellten Theorien lassen sich zur Operationalisierung des Beziehungskontextes vier zentrale Einflussfaktoren identifizieren. Die soziale Austauschtheorie begründet die Einflussfaktoren Commitment des Kunden, Relational Social Norms, sowie die Kooperationszufriedenheit. Der Konflikttheorie soll durch die Messung der Konfliktintensität und ihrer Beziehungen zu den Relational Social Norms sowie der Kooperationszufriedenheit Rechnung getragen werden. Die einzelnen Faktoren werden im Folgenden näher charakterisiert.

\subsubsection{Commitment des Kunden}

Commitment beschreibt eine starke innere Bindung an die Geschäftsbeziehung, die durch die Facetten Stabilität sowie Opferbereitschaft gekennzeichnet ist (Anderson und Weitz 1992, S. 19, Morgan und Hunt 1994, S. 23, zu einem Überblick Pedell 2000). Dwyer et al. beschreiben es als ,implict or explict pledge of relational continuity between exchange partner" (Dwyer et al. 1987, S. 19). Commitment entsteht durch bewusst getätigte Ressourcenallokationen, die nicht mehr oder nur teilweise rückgängig gemacht werden können und sich damit in einer hohen gegenseitigen Abhängigkeit äußern (Williamson 1983, S. 519ff., Dyer und Singh 1998, S. 662ff., Mudambi und Helper 1998, S. 776, Cooper und Slagmulder 2004, S. 11ff.). Dies kann 
auch durch Werte (z. B. durch Vertrauen oder Loyalität) von Personen erreicht werden (Söllner 2001, S. 222). Es ist essenziell für eine Geschäftsbeziehung und nimmt über den Zeitverlauf zu, dabei legt es den Grundstein für die Entwicklung von Beziehungsnormen und wirkt opportunistischem Verhalten entgegen (Gundlach et al. 1995, S. 78f.). Als wichtigste Outputgröße nennt Söllner (2001) den Beziehungserfolg bzw. die Beziehungsqualität (Söllner 2001, S. 222). Liegt ein hohes Commitment vor, sind die Informationssysteme von OEM-Hersteller und Lieferant stark verknüpft, der Hersteller bemüht sich aktiv um die kooperative Verbesserung von Kostenstrukturen bei seinem Lieferanten, ohne dabei primär auf seinen Vorteil abzuzielen. Die Messung von Commitment gegenüber dem Hauptkunden basiert auf einer von Morgan und Hunt entwickelten Messskala (Morgan und Hunt 1994 S. 36).

\subsubsection{Relational Social Norms}

Zusätzlich zum Commitment ist häufig die Entstehung von sogenannten Relational Social Norms zu beobachten. Diese informellen Regeln der Zusammenarbeit bilden sich im Laufe der Kooperation heraus, werden von allen Partnern geteilt und umfassen auch Erwartungen daran, wie sich Kooperationspartner in Problemsituationen verhalten (vgl. grundlegend zur Social Embeddedness von Beziehungen Granovetter 1985). Für eine langfristige und stabile Beziehung sind geteilte Verhaltensnormen unabdingbar, um Unsicherheiten, Konflikten und opportunistischem Verhalten entgegen zu wirken (Langfield-Smith und Smith 2003, S. 286, Jap und Ganesan 2000, S. 230f., Gundlach et al. 1995, S. 81). Sie dienen primär dazu, das in Kooperationen vorherrschende Risiko der einseitigen Defektion eines Partners zu verhindern und das Vertrauen zwischen den Partnerunternehmen zu steigern. Im Gegensatz zu Commitment, das eher auf die Erwartung in der Zukunft fokussiert ist, beziehen sich Beziehungsnormen auf die Erwartungen bezüglich des Verhaltens der beteiligten Partner in einer fortwährenden Beziehung (Heide und John 1992, S. 34, Jap und Ganesan 2000, S. 230, Gundlach 1995, S. 81). Im Rahmen dieses Beitrags soll unter dem Konstrukt die Bedeutung und der Nutzen der Beziehung sowie das Verhalten der Parteien in Problemsituationen verstanden werden (Jap 2001, S. 33, Gundlach 1995, S. 90f.). Existieren zuverlässige Sozialnormen, sinkt meist auch die Konfliktintensität. Die Beziehungsnormen determinieren damit die Konfliktintensität und die Zufriedenheit der Partner mit der Kooperation.

\subsubsection{Konfliktintensität}

Als Konflikt wird allgemein ein Interessengegensatz zwischen Personen oder Gruppen bezeichnet, der aus unvereinbaren Zielen oder Vorstellungen über die Zielverwirklichung oder aus einer differenzierten Wahrnehmung der Realität resultieren kann (Kuhlmann 2001, S. 802). Konflikte entstehen, wenn eine Partei der Meinung ist, dass ihre Interessen oder ihre Ziele negativ von der anderen Partei beeinflusst werden (Anderson und Narus 1984, S. 65, Kumar et al. 1995, S. 350). Die Konfliktintensität bezieht sich damit auf die Intensität der Meinungsverschiedenheit zwischen den Partnern (Palmatier et al. 2006, S. 138). Das Messinstrument basiert auf einer von Jap und Ganesan entwickelten Skala (Jap und Ganesan 2000, S. 243). 


\subsubsection{Kooperationszufriedenheit und Erfolg}

Der Erfolg wird in dieser Untersuchung aufgrund des Untersuchungsdesigns mit zwei Konstrukten gemessen: Einerseits wird der wirtschaftliche (Gesamt-) Erfolg des Zulieferers gemessen, andererseits findet die Kooperationszufriedenheit Berücksichtigung. Sie stellt eine direkte Outputgröße in der untersuchten Geschäftsbeziehung dar, da die Befragung in den Beziehungskonstrukten jeweils auf die Geschäftsbeziehung des Zulieferers mit dem größten Umsatz abstellte. Kooperationszufriedenheit wird hier verstanden als „... positive affective state resulting from the appraisal of all aspects of a firm's working relationship with another firm" (Anderson und Narus 1986, S. 326). Eine hohe Zufriedenheit mit der Geschäftsbeziehung führt zu einer langfristigen Fortführung der Beziehung (Anderson und Narus 1990, S. 46). „Beziehungserfolg beschreibt das eigentliche Ziel, das eine Partei in einer Geschäftsbeziehung verfolgt, nämlich die Lösung eines bestimmten Problems." (Söllner 2001, S. 222). Dieser betrifft damit die Qualität bzw. Stärke der Zusammenarbeit mit dem Kooperationspartner und die Wahrnehmung des finanziellen Erfolgs (Palmatier et al. 2006, S. 138). Der Beziehungserfolg wird über eine von Jap und Ganesan (2000) entwickelte Skala gemessen (Jap und Ganesan 2000, S. 243). Der finanzielle Erfolg dagegen bezieht sich auf den Gewinn und den Umsatz, der durch die Zusammenarbeit mit dem Kunden erwirtschaftet wird (Jap 1999, S. 466).

\subsection{Ableitung des Untersuchungsmodells}

Im Folgenden sollen die Beziehungen zwischen den einzelnen Konstrukten und damit die Hypothesen des Untersuchungsmodells eingeführt werden. Abbildung 1 gibt dazu einen Überblick.

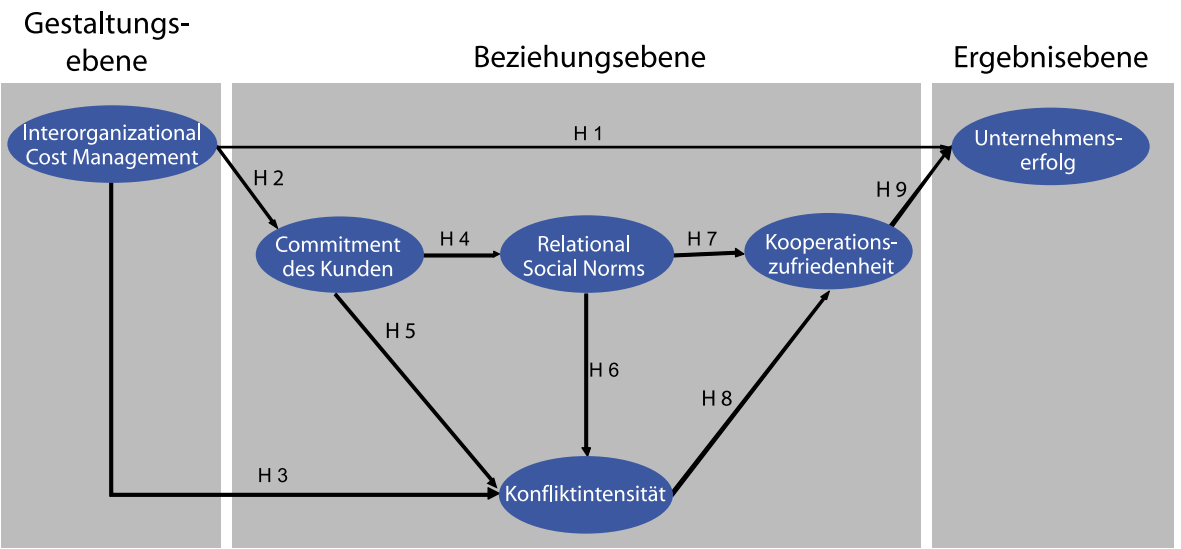

Abb. 1 Untersuchungsmodell

$\mathrm{Zu}$ Beginn soll der direkte Zusammenhang von IOCM und dem Unternehmenserfolg überprüft werden, wie er in der Literatur vorgeschlagen wird (Mouritsen et al. 
2001, S. 222, Cooper und Slagmulder 1999, S. 145f., Corsten und Kumar 2005, S. 78):

\section{H1: Der Einsatz, von IOCM steigert den Unternehmenserfolg.}

Wie bereits eingangs angesprochen und theoretisch fundiert, erscheint die Betrachtung der direkten Beziehung zwischen IOCM und Unternehmenserfolg zu kurz gegriffen. Daher ist die Berücksichtigung des Beziehungskontextes notwendig, der den Erfolg des IOCM determiniert. Folglich steht im Mittelpunkt dieses Beitrags die empirische Überprüfung, inwieweit der Erfolg des IOCM vom Beziehungskontext abhängt. Dieser wird in den Hypothesen $\mathrm{H} 2$ bis $\mathrm{H} 9$ untersucht, die Gegenstand der folgenden Ausführungen sind. Eingenommen wird dabei die Perspektive des Zulieferers.

Für den effizienten Einsatz von IOCM ist eine weitgehende Offenlegung intimer Kosteninformationen erforderlich, jedoch steigt dadurch auch die Gefahr opportunistischer Ausnutzung. Diese Gefahr lässt sich durch ein hohes Commitment des OEMHerstellers begrenzen, das hier auch die Funktion eines Signaling gegenüber dem Lieferanten übernimmt. Um den (schwächeren) Lieferant zur Preisgabe seiner Daten und zu einer offenen Kooperation bzgl. einer Kostenoptimierung zu bewegen, muss der OEM glaubwürdige Selbstbindungen (,credible commitments“, Williamson 1983) erbringen. Nur wenn er dem Lieferanten entsprechende Signale übermittelt und diese einhält, kann er mit einer Kooperation rechnen (Jap und Ganesan 2000, S. 233, Gundlach et al. 1995, S. 79; Dwyer et al. 1987, S. 19). Daraus folgt:

H2: Je stärker das IOCM ausgeprägt ist, desto höher ist das Commitment des OEMHerstellers zu seinem Lieferanten.

Die Offenlegung intimer Kosteninformationen birgt allerdings auch ein Konfliktpotenzial, da die beteiligten Partner sich dann ein besseres Urteil bilden können, inwieweit die bisher veranschlagten Preise der Lieferanten und Hersteller ,fair“ waren oder sich aufgrund eines ungleichen Machtverhältnisses zwischen Hersteller und Lieferant ergeben haben. Es kommt zu Verteilungs- und Machtkonflikten. Des Weiteren kann die Einführung von IOCM durch die Veränderung der Aufgabenverteilung zu Rollenkonflikten führen, da Rollenerwartungen wie die des Vertrauens auf Geheimhaltung oder kooperatives Verhalten seitens des Herstellers verletzt werden könnten. Zudem besteht die Möglichkeit, dass mit der Offenlegung von Kosteninformationen verbundene, gesteigerte ,Verwundbarkeit“ der beteiligten Partnerunternehmen die jeweiligen Machtgrundlagen der Partner verändert und so neue Konflikte hervorgerufen werden könnten. Entsprechend lässt sich erwarten:

\section{H3: Der Einsatz von IOCM steigert die Konfliktintensität.}

Ein hohes Commitment des OEM-Herstellers begünstigt die Entstehung zuverlässiger Beziehungsnormen, die sich mit zunehmender Länge der Interaktion zwischen den Partnern herausbilden. Der Grad der sozialen Einbettung ist daher vom Commitment des OEM-Herstellers abhängig (Gundlach et al. 1995, S. 81, 90). Die mit dem Commitment verbundenen, spezifischen Investitionen in die Partnerschaft und die damit einhergehende Abhängigkeit fördern die Wirksamkeit der Beziehungsnormen (Gundlach et al. 1995, S. 79f.), so dass sich vermuten lässt: 
H4: Je stärker das Commitment des Kunden, desto stärker sind die Beziehungsnormen ausgeprägt.

Das Commitment des OEM-Herstellers beinhaltet die Zusage einer vertrauensvollen, nicht ausschließlich auf den eigenen Vorteil bedachten Zusammenarbeit, deren Glaubwürdigkeit durch entsprechende Investitionen gefördert wird. Die höhere resultierende Beziehungsqualität führt dann zu einer niedrigeren Konfliktintensität (Jap und Ganesan 2000, S. 233):

H5: Je stärker das Commitment des Kunden, desto niedriger ist die Konfliktintensität.

Die Beziehungsnormen beinhalten auch informelle Regeln, wie Konflikte gehandhabt oder gelöst werden können. Sie determinieren damit die Konfliktintensität. Darüber hinaus wird das Verhalten der Partner durch etablierte informelle Regeln vorhersagbarer, das Vertrauen zwischen den Partnern steigt und das Risiko opportunistischer Ausnutzung sinkt. Bereits die Zusammenarbeit an sich kann nur gelingen, wenn die Zielkonflikte nicht zu hoch und die Vertrauenswürdigkeit im Sinne erkennbarer kooperationsförderlicher Intention vorhanden sind (Bierhoff 1991, S. 27ff.). Liegen starke Beziehungsnormen vor, sinkt meist auch die Konfliktintensität (Zaheer et al. 1998, S. 145), so dass sich folgern lässt:

H6: Je stärker die Beziehungsnormen, desto geringer die Konfliktintensität.

Zuverlässige Beziehungsnormen senken das Opportunismusrisiko und stellen darüber hinaus sicher, dass eine vertrauensvolle Interaktion zwischen den Partnern stattfindet. Die Partnerunternehmen empfinden die Zusammenarbeit als ausgeglichen und fair, die Zufriedenheit der Partner mit der Kooperation steigt (Skinner et al. 1992, S. 188). Daraus folgt:

\section{H7: Je stärker die Beziehungsnormen, desto höher die Kooperationszufriedenheit.}

Im Gegensatz dazu führt eine hohe Konfliktintensität zu einer geringen Kooperationszufriedenheit, da die Ziele der Kooperation, eine gemeinsame Leistung kooperativ zu erstellen, durch Konflikte behindert werden. Führt man die Konflikte auf die Wahrnehmung einer unfairen Preisbildung innerhalb der Kooperation zurück, ergibt sich ebenfalls eine niedrigere Kooperationszufriedenheit. Konflikte wirken sich daher negativ auf die Kooperationszufriedenheit aus (Skinner et al. 1992, S. 185, 187), so dass folgendes gilt:

\section{H8: Je höher die Konfliktintensität, desto geringer die Kooperationszufriedenheit.}

Als letzter Baustein lässt sich die Kooperationszufriedenheit mit dem Unternehmenserfolg verbinden. Die an der Kooperation beteiligten Partnerunternehmen sind primär dann mit der Kooperation zufrieden, wenn deren jeweiligen Ziele erreicht werden. Mit der Zielerreichung der Kooperation, deren Ziele sich meist aus dem Ziel des Unternehmenserfolgs der Partnerunternehmen ableiten, ist damit eine Erhöhung des Unternehmenserfolgs wahrscheinlich.

H9: Je höher die Kooperationszufriedenheit, desto höher der Unternehmenserfolg.

Die Hypothesen H2-H9 bilden den „Umweg“ vom Einsatz des IOCM zum Unternehmenserfolg über die Beziehungsebene, sie stellen damit eine differenziertere Betrachtung der Zusammenhänge von IOCM und Unternehmenserfolg dar. 


\section{Ergebnisse der empirischen Untersuchung}

\subsection{Informationen zum verwendeten Datensatz}

Das Hypothesensystem wurde im Rahmen einer großzahligen empirischen Studie untersucht. Die Datenerhebung erfolgte in Form eines vollstandardisierten, schriftlichen Fragebogens, der im Vorfeld ausführlichen Pre-Tests unterzogen wurde. Das Untersuchungsdesign erlaubte eine Vollerhebung, bei der sämtliche Automobilzulieferer in Deutschland auf der Basis von Branchendaten identifiziert wurden $(\mathrm{N}=733$ Unternehmen). Die Befragung erfolgte im Zeitraum September/Oktober 2006 und richtete sich an Schlüsselinformanten (Leiter Controlling/Geschäftsführung), die persönlich adressiert wurden (Zhao und Cavusgil 2006, S. 408). Die Konstrukte wurden durch geschlossene Fragen mit einer siebenstufigen Likert-Skala gemessen (Messergebnisse im Anhang). Es wurden Maßnahmen zur Erhöhung der Rücklaufquote (telefonische Rückfragen) durchgeführt. Insgesamt konnten $n=164$ Fragebögen berücksichtigt werden, was einer verhältnismäßig hohen Rücklaufquote von 22,2\% entspricht. Überprüfungen $\mathrm{zu}$ einem Non-Response-Bias und zu möglichen Verzerrungen der Stichprobe (Teilespektrum, Unternehmensgröße etc.) gegenüber der Grundgesamtheit zeigten keine Auffälligkeiten, so dass von einer hohen Repräsentativität ausgegangen werden kann. Abbildung 2 zeigt einen Überblick ausgewählter Charakteristika der Stichprobe. Die meisten der in der Studie betrachteten Zulieferbeziehungen existieren bereits seit mehr als 10 Jahren und zeigen damit einerseits eine große Stabilität, anderseits aber auch eine hohe Abhängigkeit (aufgrund der geringen OEM-Zahl). Die Umsatzverteilung ist repräsentativ für die Branche, lediglich sehr große und sehr kleine Unternehmen sind leicht unterrepräsentiert. Mehr als die Hälfte aller Zulieferer beliefern direkt die OEM-Hersteller (1st-tier), ein weiteres Drittel beliefert Direktzulieferer und den OEM, nur 10\% sind 3rd-tierLieferanten.

\subsection{Untersuchungsmodell}

Zur Beurteilung des entwickelten Untersuchungssystems wurden lokale und globale Gütemaße herangezogen. Als lokale Gütekriterien der Konstruktmessung wurden Reliabilität und Validität herangezogen. Die dazu ermittelten Werte für Cronbachs Alpha, erklärte Varianz und Faktorreliabilität weisen gute bis sehr gute Ergebnisse auf (vgl. die Angaben im Anhang). Das hier untersuchte Modell weist folgende Ausprägungen hinsichtlich der Gütemaße zur Evaluation für Strukturgleichungsmodelle auf (Homburg und Baumgartner 1998, S. 360): Goodness of Fit Index (GFI): 0,91, Adjusted Goodness of Fit Index (AGFI): 0,87, Root Mean Square Error of Approximation (RMSEA): 0,05, Comparative Fit Index (CFI): 0,96. Bis auf den AGFI weisen alle Gütemaße gute bis sehr gute Werte auf. Aufgrund der insgesamt guten Globalindices erscheint die geringe Unterschreitung des AGFI für die positive Beurteilung nicht hinderlich. Positiv zu vermerken ist das hohe Signifikanzniveau der bestätigten Korrelationen, das überwiegend auf einem Konfidenzniveau von 99,9\% liegt. Somit kann von einer guten Modellanpassung an die empirischen Daten ausgegangen werden. 


\section{Zuliefersegment}
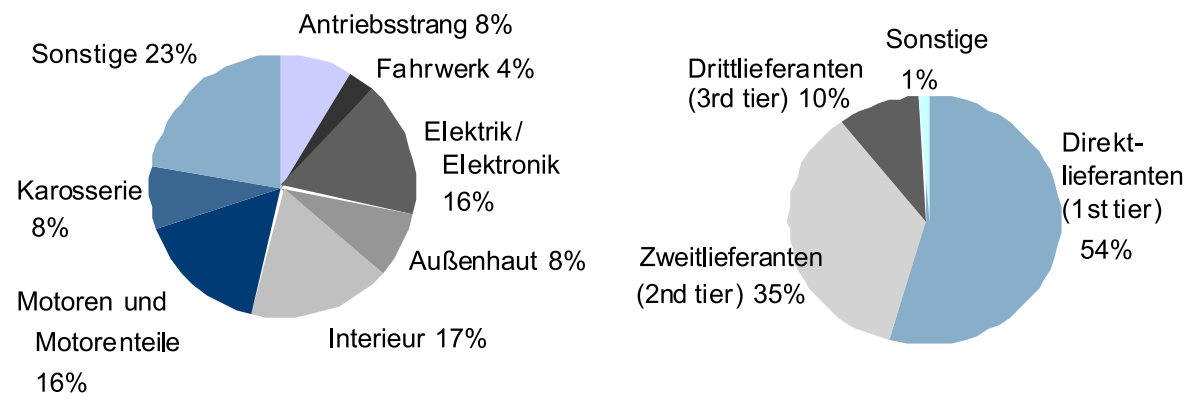

Dauer der Zulieferbeziehung

\section{Umsatzverteilung}
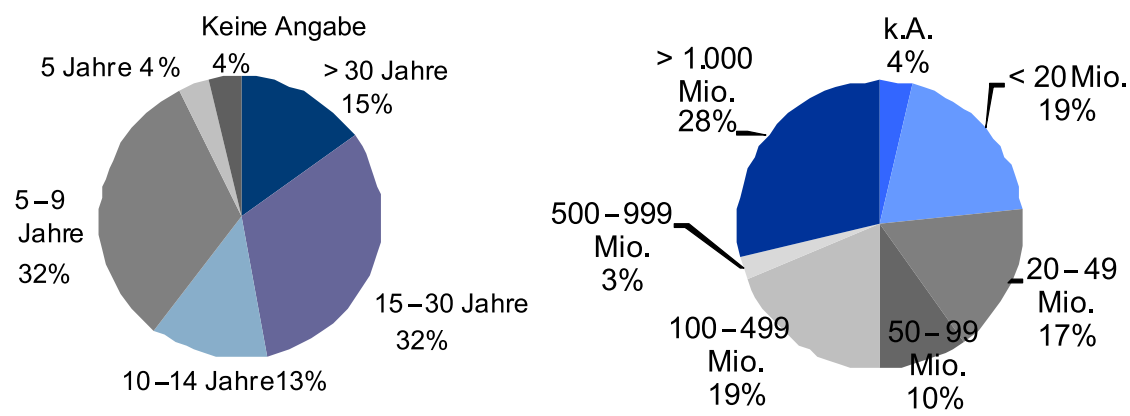

Abb. 2 Ausgewählte Charakteristika der Stichprobe

Eine direkte Beziehung zwischen dem Einsatz von IOCM und einem höheren Unternehmenserfolg konnte nicht signifikant nachgewiesen werden (H1). Dagegen ergaben sich durchgängig hohe Korrelationen und Signifikanzniveaus für den alternativen Hypothesenzug IOCM $\rightarrow$ Commitment $\rightarrow$ Relational Social Norms $\rightarrow$ Kooperationszufriedenheit $\rightarrow$ Unternehmenserfolg (H2, H4, H7, H9). Auch zwischen IOCM und der Konfliktintensität ergab sich ein mittlerer, signifikanter Zusammenhang (H3). Demgegenüber konnte die unterstellte konfliktmindernde Wirkung des Kundencommitment nicht nachgewiesen werden (H5), erforderlich scheint vielmehr die Etablierung zuverlässiger Beziehungsnormen (H6). Nachgewiesen werden konnte auch eine zufriedenheitsmindernde Wirkung der Konfliktintensität (H8) auf einem mittleren Niveau.

\subsection{Diskussion}

Es lässt sich konstatieren, dass IOCM in der Automobilzulieferindustrie eine heterogene Verbreitung aufweist. Die verwendeten Items weisen Durchschnittswerte von 3,7 bis 5,0 (auf einer Skala von 1-schwach bis 7-stark) bei einer tendenziell rechtssteilen Verteilung auf. Partiell wird IOCM offensichtlich stark praktiziert. Mithilfe der 


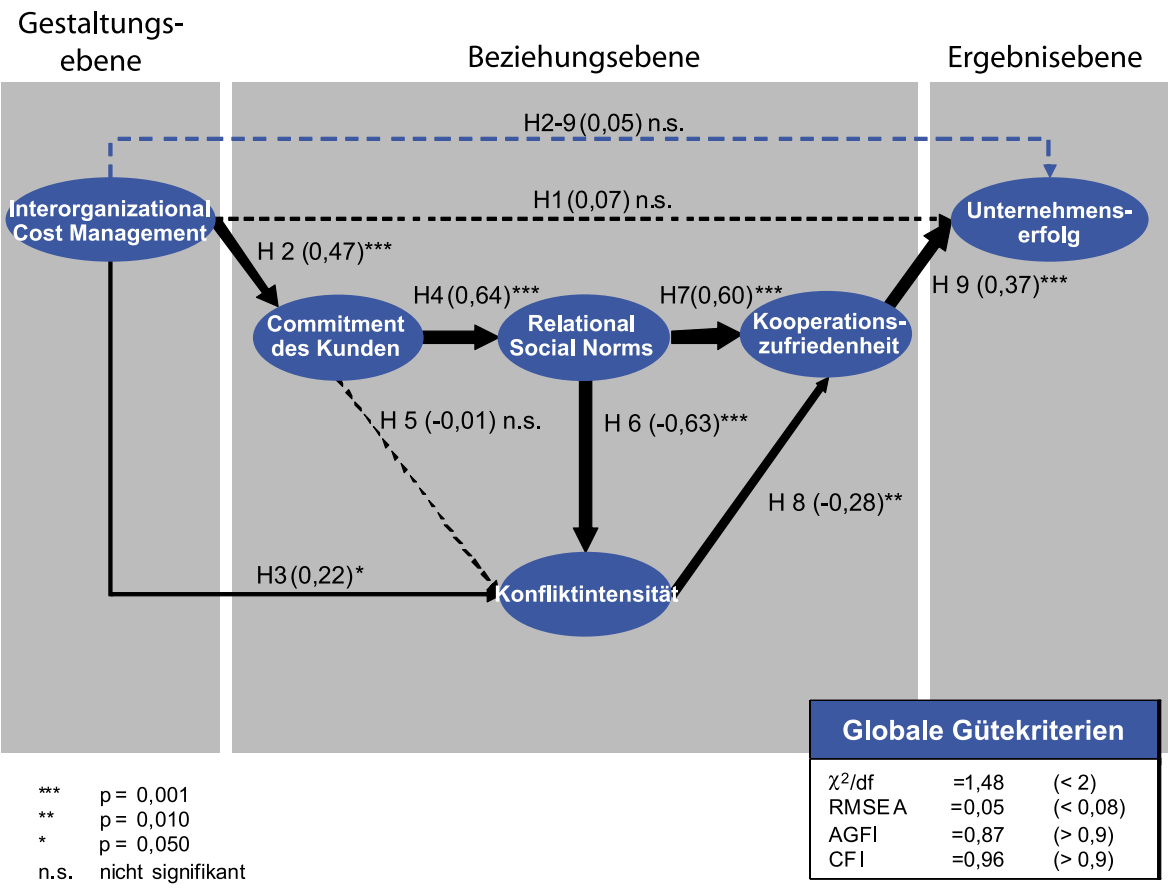

Abb. 3 Ergebnisse des Modells (die Dicke der Pfeile symbolisiert die Stärke der Korrelationen)

Ergebnisse lässt sich die titelgebende Fragestellung nun beantworten: IOCM wirkt konfliktsteigernd und kann erfolgssteigernd wirken - allerdings nur unter bestimmten Voraussetzungen. Dies soll im Folgenden näher analysiert werden.

Als Voraussetzungen für eine hohe Beziehungszufriedenheit und einen hohen finanziellen Erfolg des Zulieferers konnten in dieser Untersuchung ein hohes Commitment des Kunden und stark ausgeprägte Verhaltens- oder Sozialnormen vorläufig bestätigt werden. Kritisch zu hinterfragen ist hier allerdings die unterstellte UrsacheWirkungsbeziehung, wie sie in Abschnitt 3.3 theoriebasiert hergeleitet wurde: Ein reverser Hypothesenzug erscheint durchaus denkbar, dass nämlich zuerst die verhaltensorientierten Bedingungen gegeben sein müssen, bevor ein IOCM überhaupt implementiert werden kann. Vor dem Hintergrund der Fragestellung erscheint dies allerdings nachrangig, da das Ziel vorrangig im Nachweis eines Zusammenhanges besteht. Die praxisorientierte Identifikation einer sinnvollen Abfolge von Implementierungsschritten erscheint damit als vielversprechender Gegenstand weiterer Forschungen.

Umgekehrt erhöht (aus Sicht der Zulieferer) ein IOCM die Konfliktintensität einer Beziehung. Dies wirkt sich über eine Senkung der Beziehungszufriedenheit auch negativ auf die Erfolg des Zulieferers aus. Interessant wäre hier sicherlich die Überprüfung der Sicht des OEM. Eine naheliegende Erklärung könnte sein, dass im Rahmen des IOCM eine einseitige Kostenoptimierung aus Sicht des OEM und ,,auf dem Rücken“ des Zulieferers stattfindet. Offensichtlich wird das IOCM seitens des Zulieferers - zumindest zu einem Teil - negativ wahrgenommen. Notwendig 
erscheint daher eine Überzeugungs- bzw. Aufklärungsarbeit seitens des OEM. Erstaunlich erscheint in diesem Zusammenhang erst einmal, dass der größte Teil der befragten Unternehmen angibt, dass sie auch langfristig eine Fortführung ihrer Zusammenarbeit mit dem OEM anstreben. Vor dem Hintergrund der oligarchischen Situation in der Automobilindustrie erscheint dies allerdings plausibel. Es lässt sich zweierlei festhalten: IOCM ist eine potenzielle Quelle von Konflikten. Bisherige Arbeiten thematisieren diesen Aspekt in viel zu geringem Maße. Es lässt sich erheblicher Forschungsbedarf konstatieren, wann und unter welchen Bedingungen derartige Konflikte entstehen und wie sie bewältigt werden können. Zweitens: In der hier untersuchten Situation scheinen die Potenziale eines IOCM keineswegs effizient genutzt zu werden, da die erfolgssteigernden Wirkungen durch Konflikte zum Teil kompensiert werden. Eine präventive Gestaltung des IOCM und ein effizientes Konfliktmanagement können hier vermutlich Erfolgspotenziale erschließen.

Dies leitet über zu der nicht bestätigten Hypothese H5, dass ein Commitment des Kunden konfliktmindernd oder -vermeidend wirken soll. Offensichtlich gelingt es den OEM in dieser Studie nicht, ihre Selbstbindung glaubwürdig genug darzustellen (credible commitment). Die in den meisten Zulieferprogrammen dargestellten programmatischen Aussagen zum partnerschaftlichen Umgang mit Lieferanten (Codes of Conduct) sind in kritischen (Konflikt-) Situationen offensichtlich nicht tragfähig genug. Nur wenn auf der Verhaltens- und Beziehungsebene das Verhältnis zwischen OEM und Lieferant bereits sehr gefestigt ist, können damit Konflikte vermieden werden und die Codes of Conduct werden akzeptiert. Es lässt sich folgern, dass neben der „,technischen“ Kostenoptimierung (die Gegenstand des klassischen IOCM-Instrumentenkataloges ist) die Herstellung einer partnerschaftlichen, vertrauensvollen Zusammenarbeit eine mindestens gleichgroße Aufmerksamkeit verdient. Ein IOCM muss sich daher in Zukunft verstärkt auch derartigen ,,weichen“ Aspekten zuwenden. Das Controlling muss entsprechende Instrumente und Qualifikationen im Rahmen eines Beziehungscontrollings bereitstellen, um neben den harten Kostensenkungszielen auch die weichen Kooperationsumstände zu steuern.

\section{Fazit}

Mit der vorliegenden Untersuchung konnte nachgewiesen werden, dass IOCM nicht durch seine pure Anwendung erfolgssteigernd wirkt, sondern dies nur unter bestimmten Bedingungen der Fall ist. Nachgewiesen werden konnte hier das Vorliegen von Commitment des Kunden sowie von Beziehungs- und Verhaltensnormen. Weiterhin konnte gezeigt werden, dass IOCM konfliktsteigernd und sich damit negativ auf die Beziehungszufriedenheit und den Erfolg auswirkt. In allen Fällen wurde die Perspektive bzw. Einschätzung des Zulieferers zugrundegelegt. Nicht nachgewiesen konnte die konfliktmindernde Wirkung von Selbstbindungsmaßnahmen (Commitment) des Kunden.

Es wurden Handlungsempfehlungen zur verbesserten Anwendung des IOCM anhand der empirischen Analysen erarbeitet: (1) Differenzierter, d.h. situationsbzw. partneradäquater Einsatz des IOCM, da es nicht immer erfolgssteigernd wirkt. 
(2) Stärkere Berücksichtigung von Verhaltensbeeinflussung und von sozialen Aspekten bei der Anwendung des IOCM. (3) Systematische Integration von präventiven und reaktiven Konfliktlösungen in das IOCM. (4) Implementierung glaubwürdiger Selbstbindungsmaßnahmen (credible commitments) im IOCM. Beispielhaft wären hier die Schaffung gemeinsamer Investitionen, die gegenseitige Offenlegung von Kosteninformationen oder eine vertragliche Abnahmeverpflichtung des OEM gegenüber dem Zulieferer zu nennen. Daraus lassen sich entsprechende Forschungsbedarfe für weitere Arbeiten ableiten. Vielversprechend erscheint darüber hinaus der zeitliche Aspekt, d.h. inwiefern IOCM zu einer Verbesserung und Vertiefung der Zusammenarbeit beiträgt oder ob eine partnerschaftliche Zusammenarbeit Voraussetzung ist. In der Folge ergibt sich die Frage, wie dann eine partnerschaftliche Zusammenarbeit erreicht werden kann.

Die vorliegende Untersuchung weist verschiedene Beschränkungen auf: Kritisch zu betrachten an den Ergebnissen ist (wie in derartigen Studien üblich) die Zeitpunktbezogenheit der Daten. Gleichfalls bedingt das Untersuchungsdesign die Gefahr, dass sich die Ergebnisse auf einseitige und subjektive Beurteilungen bei der Fragebogenbeantwortung beziehen können. Interessant wäre im vorliegenden Fall sicherlich eine Kreuzvalidierung gewesen, d. h. die gleichzeitige Befragung der OEM. Aus Vertraulichkeitsgründen konnte dies in der vorliegenden Studie allerdings nicht realisiert werden, stellt aber gleichfalls eine zentrale Anregung für weitere Forschungen auf diesem Feld dar. Darüber hinaus existieren zahlreiche weitere Gestaltungsfaktoren, die in der Studie nicht berücksichtigt werden konnten, ebenso wie mögliche Effekte moderierender Variablen.

Open Access Dieser Artikel wird zu den Bedingungen der „Creative Commons Attribution Noncommercial License" zur Verfügung gestellt. Damit ist eine nichtkommerzielle Nutzung, Verbreitung und Vervielfältigung erlaubt, sofern die Autoren des Artikels und die genaue Quelle angegeben sind. 


\section{Anhang}

\section{Konstrukt: IOCM}

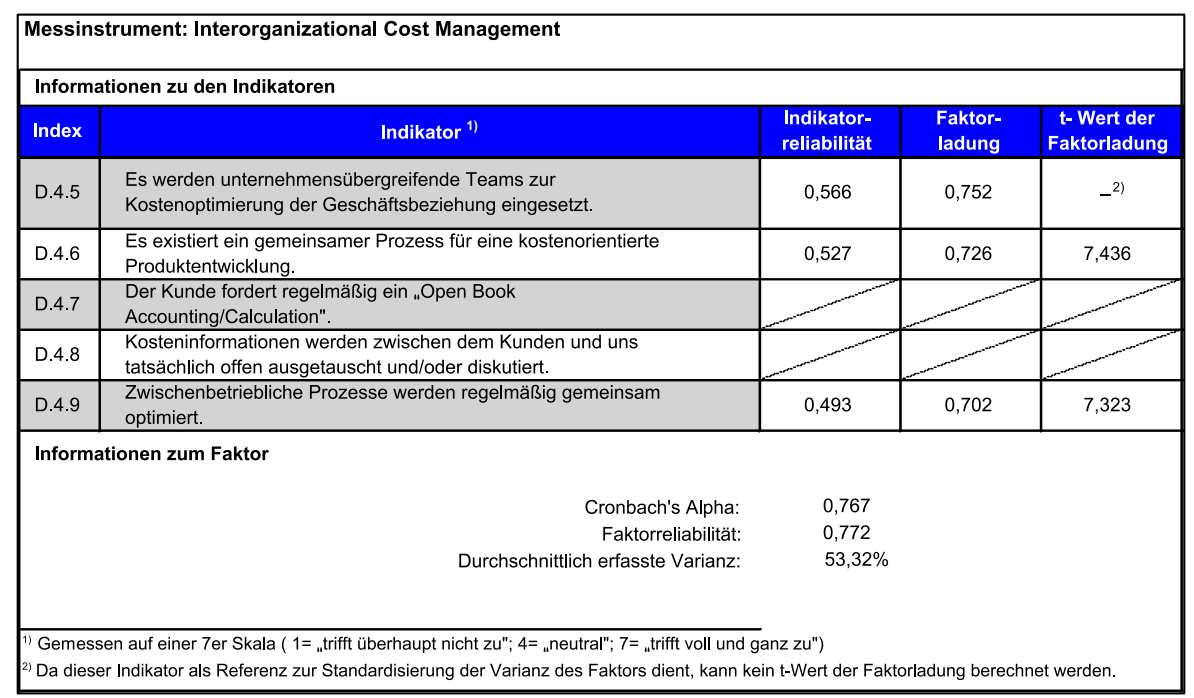

\section{Konstrukt: Commitment des Kunden}

\begin{tabular}{|c|c|c|c|c|c|}
\hline \multicolumn{6}{|c|}{ Messinstrument: Commitment des Kunden } \\
\hline \multicolumn{6}{|c|}{ Informationen zu den Indikatoren } \\
\hline Index & Indikator 1) & $\begin{array}{l}\text { Indikator- } \\
\text { reliabilität }\end{array}$ & $\begin{array}{l}\text { Faktor- } \\
\text { ladung }\end{array}$ & $\begin{array}{c}\text { t-Wert der } \\
\text { Faktorladung }\end{array}$ & $\mathbf{p}$ \\
\hline D.5.4 & $\begin{array}{l}\text { Unser Kunde hilft uns mit seinen Mitarbeitern und Ressourcen, } \\
\text { unseren Umsatz deutlich zu steigern. }\end{array}$ & 0,478 & 0,691 & $-2)$ & \\
\hline D.5.5 & $\begin{array}{l}\text { Unser Kunde investiert mehr Zeit und Mühe in uns als in } \\
\text { vergleichbare andere Zulieferer. }\end{array}$ & 0,525 & 0,724 & 7,393 & $* * *$ \\
\hline D.5.6 & $\begin{array}{l}\text { Unser Kunde kommt uns regelmäßig bei Vereinbarungen } \\
\text { entgegen. }\end{array}$ & 0,560 & 0,748 & 7,668 & *** \\
\hline D.5.7 & Unser Kunde ist geduldig mit uns, wenn wir Fehler machen. & & & & \\
\hline D.5.8 & $\begin{array}{l}\text { Unser Kunde hält Ausschau nach anderen Zulieferern, um uns zu } \\
\text { ersetzen. (R) }\end{array}$ & & & & \\
\hline \multicolumn{6}{|c|}{ Informationen zum Faktor } \\
\hline & $\begin{array}{r}\text { Cronbach's Alpha: } \\
\text { Faktorreliabilität: } \\
\text { Durchschnittlich erfasste Varianz: }\end{array}$ & $\begin{array}{l}0,766 \\
0,761 \\
51,64 \%\end{array}$ & & & \\
\hline \multicolumn{6}{|c|}{$\begin{array}{l}\text { 1) Gemessen auf einer } 7 \text { er Skala ( } 1=\text { „trifft überhaupt nicht zu”; } 4=\text { „neutral”; } 7=\text { „trifft voll und ganz zu”) } \\
\text { 2) Da dieser Indikator als Referenz zur Standardisierung der Varianz des Faktors dient, kann kein t-Wert der Faktorladung berechnet werden. } \\
\text { (R) = Reverse-Coded Item, d.h. umgekehrte Skala im Vergleich zu den anderen Indikatoren }\end{array}$} \\
\hline
\end{tabular}




\section{Konstrukt: Beziehungsnormen}

\begin{tabular}{|c|c|c|c|c|c|}
\hline \multicolumn{6}{|c|}{ Messinstrument: Beziehungsnormen } \\
\hline \multicolumn{6}{|c|}{ Informationen zu den Indikatoren } \\
\hline Index & Indikator') & $\begin{array}{l}\text { Indikator- } \\
\text { reliabilität }\end{array}$ & $\begin{array}{l}\text { Faktor- } \\
\text { ladung }\end{array}$ & $\begin{array}{c}\text { t- Wert der } \\
\text { Faktorladung }\end{array}$ & $\mathbf{p}$ \\
\hline D.7.1 & $\begin{array}{l}\text { Die Zusammenarbeit ist für beide Unternehmen } \\
\text { auch im Angesicht von Problemen und } \\
\text { Herausforderungen sehr wichtig. }\end{array}$ & & & & \\
\hline D.7.2 & Die Beziehung basiert auf beiderseitigen Vorteilen. & & & & \\
\hline D.7.3 & Die Beziehung basiert auf beiderseitigem Vertrauen. & 0,446 & 0,668 & $-^{2)}$ & *** \\
\hline D.7.4 & $\begin{array}{l}\text { Die Zusammenarbeit ist entgegenkommend, wenn } \\
\text { Probleme auftreten. }\end{array}$ & 0,799 & 0,894 & 9,666 & *** \\
\hline D.7.5 & Ein Informationsaustausch findet häufig statt. & 0,763 & 0,874 & 9,602 & 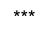 \\
\hline \multicolumn{6}{|c|}{ Informationen zum Faktor } \\
\hline \multicolumn{2}{|r|}{$\begin{array}{r}\text { Cronbach's Alpha: } \\
\text { Faktorreliabilität: }\end{array}$} & $\begin{array}{l}0,847 \\
0,854 \\
66,28 \%\end{array}$ & & & \\
\hline \multicolumn{6}{|c|}{ 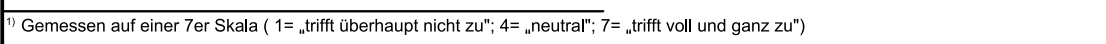 } \\
\hline
\end{tabular}

\section{Konstrukt: Beziehungszufriedenheit}

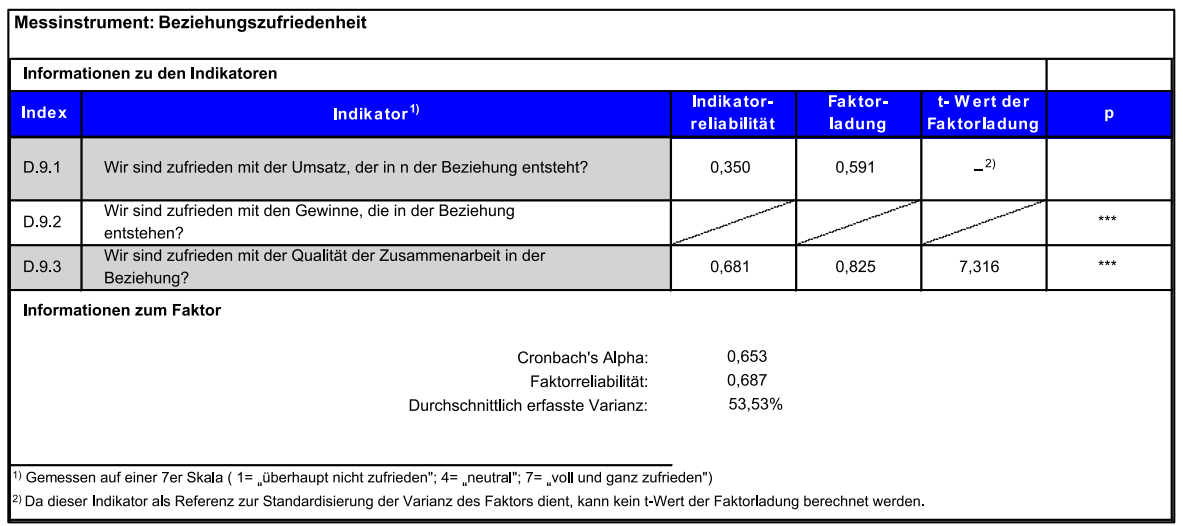




\section{Konstrukt: Konfliktintensität}

\begin{tabular}{|c|c|c|c|c|c|}
\hline \multicolumn{6}{|c|}{ Messinstrument: Konfliktintensität } \\
\hline \multicolumn{6}{|c|}{ Informationen zu den Indikatoren } \\
\hline Index & Indikator ${ }^{1)}$ & $\begin{array}{l}\text { Indikator- } \\
\text { reliabilität }\end{array}$ & $\begin{array}{l}\text { Faktor- } \\
\text { ladung }\end{array}$ & $\begin{array}{c}\text { t-Wert der } \\
\text { Faktorladung }\end{array}$ & p \\
\hline D.8.1 & Die Geschäftsbeziehung kann als angespannt bezeichnet werden. & 0,712 & 0,844 & 13,352 & \\
\hline D.8.2 & Wir haben starke Meinungsverschiedenheiten in unserer Beziehung. & 0,871 & 0,933 & 15,068 & *** \\
\hline D.8.3 & Wir haben regelmäßig Differenzen bzgl. unserer Zusammenarbeit. & 0,714 & 0,845 & $-2)$ & *** \\
\hline \multicolumn{6}{|c|}{ Informationen zum Faktor } \\
\hline & $\begin{array}{r}\text { Cronbach's Alpha: } \\
\text { Faktorreliabilität: } \\
\text { Durchschnittlich erfasste Varianz: }\end{array}$ & $\begin{array}{l}0,905 \\
0,906 \\
76,31 \%\end{array}$ & & & \\
\hline 1) Gemes & $\begin{array}{l}\text { auf einer 7er Skala ( } 1=\text { „trifft überhaupt nicht zu"; } 4={ }_{\text {" }} \text { neutral"; } 7=\text { "trifft voll und ganz } \\
\text { dikator als Referenz zur Standardisierung der Varianz des Faktors dient, kann kein t- }\end{array}$ & der Faktorladur & hnet & & \\
\hline
\end{tabular}

\section{Konstrukt: Unternehmenserfolg}

\begin{tabular}{|c|c|c|c|c|c|}
\hline \multicolumn{6}{|c|}{ Messinstrument: Relativer Unternehmenserfolg } \\
\hline \multicolumn{6}{|c|}{ Informationen zu den Indikatoren } \\
\hline Index & Indikator ${ }^{1)}$ & $\begin{array}{l}\text { Indikator- } \\
\text { reliabilität }\end{array}$ & $\begin{array}{l}\text { Faktor- } \\
\text { ladung }\end{array}$ & $\begin{array}{c}t \text { - Wert der } \\
\text { Faktorladung }\end{array}$ & p \\
\hline E.1.1 & Marktanteil & 0,638 & 0,799 & $-{ }^{2)}$ & \\
\hline E.1.2 & Umsatz & 0,939 & 0,969 & 11,007 & *** \\
\hline E.1.3 & Gewinn & 0,380 & 0,616 & 8,350 & *** \\
\hline E.1.4 & Schwankung des Gewinns & & & & \\
\hline \multicolumn{6}{|c|}{ Informationen zum Faktor } \\
\hline & $\begin{array}{r}\text { Cronbach's Alpha: } \\
\text { Faktorreliabilität: } \\
\text { Durchschnittlich erfasste Varianz: }\end{array}$ & $\begin{array}{l}0,826 \\
0,830 \\
62,49 \%\end{array}$ & & & \\
\hline $\begin{array}{l}\text { Gemes } \\
\text { Da dies }\end{array}$ & $\begin{array}{l}\text { auf einer 7er Skala ( } 1 \text { = „schwach unterdurchschnittlich"; 4= „durchschnittli } \\
\text { dikator als Referenz zur Standardisierung der Varianz des Faktors dient, }\end{array}$ & $=$ "stark überd & hnittlich") & & \\
\hline
\end{tabular}

\section{Literatur}

Adler, P.S.: Market, hierarchy and trust: the knowledge economy and the future of capitalism. Organ. Sci. 12(2), 215-234 (2001)

Anderson, S.W.: Managing Costs and Cost Structures throughout the Value Chain: Research on Strategic Cost Management. In: Chapmann, C.S., Hopwood, A.D., Shields, M.D. (eds.) Handbook of Management Accounting Research, pp. 481-506. Elsevier, Oxford (2007)

Anderson, E., Weitz, B.: The use of pledges to build and sustain commitment in distribution channels. J. Mark. Res. 29(1), 18-34 (1992)

Anderson, J.C., Narus, J.A.: A model of the distributor's perspective of distributor-manufacturer working relationships. J. Mark. 48, 62-74 (1984)

Anderson, J.C., Narus, J.A.: Toward a Better Understanding of Distribution Channel Working Relationships. In: Backhaus, K., Wilson, D. (eds.) Industrial Marketing, pp. 320-326. Springer, Berlin (1986) 
Anderson, J.C., Narus, J.A.: A model of distributor firm and manufacturer firm working partnerships. J. Mark. 54(1), 42-58 (1990)

Balling, R.: Kooperation: Strategische Allianzen, Netzwerke, Joint-Ventures und andere Organisationsformen zwischenbetrieblicher Zusammenarbeit in Theorie und Praxis. Lang, Frankfurt (1997)

Beutin, N.: Kundennutzen in industriellen Geschäftsbeziehungen. Gabler, Wiesbaden (2000)

Bierhoff, H.W.: Soziale Motivation kooperativen Verhaltens. In: Wunderer, R. (Hrsg.) Kooperation: Gestaltungsprinzipien und Steuerung der Zusammenarbeit zwischen Organisationseinheiten, S. 21-38. Poeschel, Stuttgart (1991)

Blau, P.: Exchange and power in social life. Transaction Publishers, New York (1964)

Carr, C., Ng, J.: Total cost control: Nissan and its U.K. supplier partnerships. Manag. Account. Res. 6, 347-365 (1995)

Coase, R.H.: The nature of the firm. Economica 4(13), 386-405 (1937)

Cooper, R., Yoshikawa, T.: Inter-organizational cost management systems: The case of the TokyoYokohama-Kamakura supplier chain. Int. J. Prod. Econ. 37(1), 51-62 (1994)

Cooper, R., Slagmulder, R.: Supply Chain Development For The Lean Enterprise: Interorganizational Cost Management. Productivity Press, Portland (1999)

Cooper, R., Slagmulder, R.: Interorganizational cost management and relational context. Account. Organ. Soc. 29(1), 1-26 (2004)

Corsten, D., Kumar, N.: Do suppliers benefit from collaborative relationships with large retailers? An empirical investigation of efficient consumer responce adoption. J. Mark. 69(3), 80-94 (2005)

Das, T.K., Teng, B.-S.: Alliance constellations: A social exchange perspective. Acad. Manag. Rev. 27(3), 445-456 (2002)

Dekker, H.: Value chain analysis in interfirm relationships: A field study. Manag. Account. Res. 14, 1-23 (2003)

Dekker, H.C.: Control of inter-organizational relationships: Evidence on appropriation concerns and coordination requirements. Account. Organ. Soc. 29(1), 27-49 (2004)

Dwyer, F.R., Schurr, P.H., Oh, S.: Developing buyer-seller relationships. J. Mark. 51(2), 11-27 (1987)

Dyer, J.H., Singh, H.: The relational view: Cooperative strategy and sources of interorganizational competitive advantage. Acad. Manag. Rev. 23(4), 660-679 (1998)

Granovetter, M.: Economis action and social structure: The problem of embedness. Am. J. Sociol. 91, 481-510 (1985)

Gundlach, G.T., Achrol, R.S., Mentzer, J.T.: The struture of commitment in exchange. J. Mark. 59(1), 78-92 (1995)

Hakansson, H., Lind, J.: Accounting in an Interorganizational Setting. In: Chapmann, C.S., Hopwood, A.D., Shields, M.D. (eds.) Handbook of Management Accounting Research, pp. 885-904. Elsevier, Oxford (2007)

Heide, J.B., John, G.: Do norms matter on marketing relationships? J. Mark. 56(2), 32-44 (1992)

Homburg, C., Baumgartner, H.: Beurteilung von Kausalmodellen: Bestandsaufnahme und Anwendungsempfehlungen. In: Hildebrandt, L., Homburg, C. (Hrsg.) Die Kausalanalyse: ein Instrument der empirischen betriebswirtschaftlichen Forschung, S. 345-369. Schäffer-Poeschel, Stuttgart (1998)

Jap, S.D.: Pie-expansion efforts collaboration processes in buyer-supplier relationships. J. Mark. Res. 36(4), 461-475 (1999)

Jap, S.D.: Perspectives on joint competitive advantages in buyer-supplier relationships. Int. J. Res. Mark. 18(1-2), 19-35 (2001)

Jap, S.D., Ganesan, S.: Control mechanisms and relationship life cycle: Implications for safeguarding specific investments and developing commitment. J. Mark. Res. 37(2), 227-245 (2000)

Kajüter, P.: Proaktives Kostenmanagement. Gabler, Wiesbaden (2000)

Kajüter, P.: Proactive cost management in supply chains. In: Seuring, S., Goldbach, M. (eds.) Cost Management in Supply Chains, pp. 31-52. Springer, Heidelberg (2002)

Kajüter, P., Franz, K.-P.: Kostenmanagement: Wertsteigerung durch systematische Kostensteuerung. Schäffer-Poeschel, Stuttgart (2002)

Kajüter, P., Kulmala, H.I.: Open-book accounting in networks: Potential achievements and reasons for failures. Manag. Account. Res. 16(2), 179-204 (2005)

Krystek, U., Redel, W., Reppegather, S.: Grundzüge virtueller Organisationen: Elemente und Erfolgsfaktoren, Chancen und Risiken. Gabler, Wiesbaden (1997)

Kuhlmann, E.: Soziale Konflikte. In: Diller, H. (Hrsg.) Vahlens Großes Marketinglexikon, 2. Aufl., S. 802. Vahlen, München (2001)

Kulmala, H.I.: Developing cost management in customer-supplier relationships: three case studies. J. Purch. Supply Manag. 10, 65-77 (2004) 
Kumar, N., Scheer, L.K., Steenkamp, J.B.: The effects of perceived interdependence on dealer attitudes. J. Mark. Res. 32(3), 348-356 (1995)

Langfield-Smith, K., Smith, D.: Management control systems and trust in outsourcing relationships. Manag. Account. Res. 14, 281-307 (2003)

Meinig, W., Heß, A.: Konflikte in vertraglichen Vertriebssystemen der Automobilwirtschaft. Z. Betriebswirtsch. 62(4), 369-390 (1992)

Möller, K.: Zuliefererintegration in das Target Costing auf Basis der Transaktionskostentheorie. Vahlen, München (2002)

Möller, K.: Wertschöpfung in Netzwerken. Vahlen, München (2006)

Morgan, R.M., Hunt, S.D.: The commitment-trust theory of relationship marketing. J. Mark. 58(3), 2038 (1994)

Mouritsen, J., Hansen, A., Hansen, C.O.: Inter-organizational controls and organizational competencies: episodes around target cost management/functional analysis and open book accounting. Manag. Account. Res. 12(2), 221-244 (2001)

Mudambi, R., Helper, S.: The 'close but adversarial' model of supplier relations in the U.S. auto industry. Strateg. Manag. J. 19(8), 775-792 (1998)

Palmatier, R.W., Dant, R.P., Grewal, D., Evans, K.R.: Factors influencing the effectiveness of relationship marketing: A meta-analysis. J. Mark. 70(4), 136-153 (2006)

Pampel, J.: Kooperation mit Zulieferern. Theorie und Management. Gabler, Wiesbaden (1993)

Pedell, B.: Commitment als Wettbewerbsstrategie. Duncker und Humblot, Berlin (2000)

Picot, A.: Transaktionskostenansatz in der Organisationstheorie: Stand der Diskussion und Aussagewert. DBW 42(2), 267-284 (1982)

Picot, A., Reichwald, R., Wigand, R.T.: Die grenzenlose Unternehmung: Information, Organisation und Management, 5. Aufl. Gabler, Wiesbaden (2003)

Picot, A., Dietl, H., Franck, E.: Organisation: Eine ökonomische Perspektive, 2. Aufl. Schäffer-Poeschel, Stuttgart (1999)

Rusbult, C.: A longitudinal test of the investment model: The impact on job satisfaction, job commitment, and turnover of variations in rewards, costs, alternatives, and investments. J. Appl. Psychol. 68(3), 429-438 (1983)

Seuring, S.: Supply Chain Costing. Vahlen, München (2001)

Seuring, S., Goldbach, M. (Hrsg.): Cost Management in Supply Chains. Springer, Heidelberg (2002)

Skinner, J.S., Gassenheimer, J.B., Kelley, S.W.: Cooperation in supplier-dealer relations. J. Retail. 68, 174-193 (1992)

Söllner, A.: Commitment. In: Diller, H. (Hrsg.) Vahlens Großes Marketinglexikon, 2. Aufl., S. 222-223. Vahlen, München (2001)

Steffenhagen, H.: Konflikt und Kooperation in Absatzkanälen: Ein Beitrag zur verhaltensorientierten Marketingtheorie. Gabler, Wiesbaden (1975)

Stölzle, W.: Industrial Relationships. Vahlen, München (1999)

Stölzle, W., Otto, A. (Hrsg.): Supply Chain Controlling in Theorie und Praxis. Gabler, Wiesbaden (2003)

Thibaut, J., Kelley, H.: The Social Psychology of Groups. Transaction Publishers, New York (1959)

Wieselquist, J., Rusbult, C.E., Foster, C.A., Agnew, C.R.: Commitment, pro-relationship behavior, and trust in close relationships. J. Pers. Soc. Psychol. 77(5), 942-966 (1999)

Williamson, O.E.: Markets and Hierarchies: Analysis and Antitrust Implications. Free Press, New York (1975)

Williamson, O.E.: Credible commitments: Using hostages to support exchange. Am. Econ. Rev. 73(4), 519-540 (1983)

Williamson, O.E.: The economic institutions of capitalism: firms, markets, relational contracting. Free Press, New York (1985)

Zaheer, A., McEvily, B., Perrone, V.: Does trust matter? Exploring the effects of interorganizational and interpersonal trust on performance. Organ. Sci. 9(2), 141-159 (1998)

Zhao, Y., Cavusgil, T.S.: The effect of supplier's market orientation on manufactuerer's trust. Ind. Mark. Manag. 35(4), 405-414 (2006) 